\title{
The Currency of Words: An Exploration of Motivational Prosody
}

\author{
Maitri Jain ${ }^{* 1}$ and Priti Dhawan² \\ ${ }^{1}$ Department of Counseling and Clinical Psychology, Teachers College, Columbia University \\ ${ }^{2}$ Department of Psychology, Lady Shri Ram College for Women, University of Delhi, Delhi, India
}

*Corresponding author: maitri.jain.mj@gmail.com

Received: 11-05-2020

Revised: $19-07-2020$

Accepted: 24-09-2020

\begin{abstract}
Prosody plays an important role in students' social and academic achievement. To explore the impact of motivational prosody on teaching effectiveness, we conducted a quasi-experimental study on ninety-three undergraduate students. Teaching effectiveness was measured through students' intrinsic motivation, task valuing, perceived choice, perceived competence, tension, effort expended, and creative problem solving. The prosodies investigated included autonomy-granting, controlling, and neutral prosodies. We used The Intrinsic Motivation Inventory (Deci \& Ryan, 2005) and Passi-Usha Test for Creative Problem Solving (Passi \& Kumar, 1996) for measuring the constructs. Statistical analysis along with the follow-up interviews revealed that the groups significantly differed in creative problem solving, with an effect size of 0.118 . Moreover, the difference in scores for creative problem solving was due to differences in level of elaboration by participants, whereas the originality of responses was same across groups. This suggests that even though prosody has a role to play, a much more complex network of interconnected factors determines the student-teacher relationship.
\end{abstract}

Keywords: Instructional practices, motivational prosody, intrinsic motivation, creative problem solving

There are well known models that list the characteristics of an effective teacher, such as McBer'sModel of Teacher Effectiveness (Fig. 1) and The Need Supportive Teaching Framework (Fig. 2).

The common factor that underlies them all, however, is the quality of verbal dialogue between the teacher and the students. The tone in which the teacher speaks, her speed of talking, the pauses she takes, all affect how positively her students will view her, and the amount of improvement in learning and behavior she would be able to inculcate. Therefore, she should communicate in the "correct" tone of voice to have a positive impact on her pupils and to develop constructive relationships with them. Tone of voice is also referred to as prosody, and helps determine the intended meaning of an utterance. It is the source of different perceptions of speech signals. Weinstein et al. (2017) conducted the groundbreaking study exploring the role prosody plays in motivating students, and found that, listeners who heard the autonomy-granting tone had higher well-being, more experience of support, and a closer emotional bond with the teacher than students in the controlling tone condition.

The Self-determination Theory (Deci \& Ryan, 1985)

How to cite this article: Jain, M. and Dhawan, P. (2020). The Currency of Words: An Exploration of Motivational Prosody. Int. J. Soc. Sci., 9(03): 117126.

Source of Support: None; Conflict of Interest: None 
eloquently explains these fascinating yet mundane findings. As a student is given more autonomy, his or her intrinsic motivation increases, leading to an increase in persistence, achievement, learning, challengeseeking, creativity, and positive emotions. Providing free choice improves individuals' intrinsic motivation, effort expended on tasks, performance on them, and their level of perceived competence. Students also rate their skills higher and are more satisfied with what they are doing if they value the task at hand. Pressure, on the other hand, negatively predicts intrinsic motivation, and further reduces self-efficacy and the quality of work.

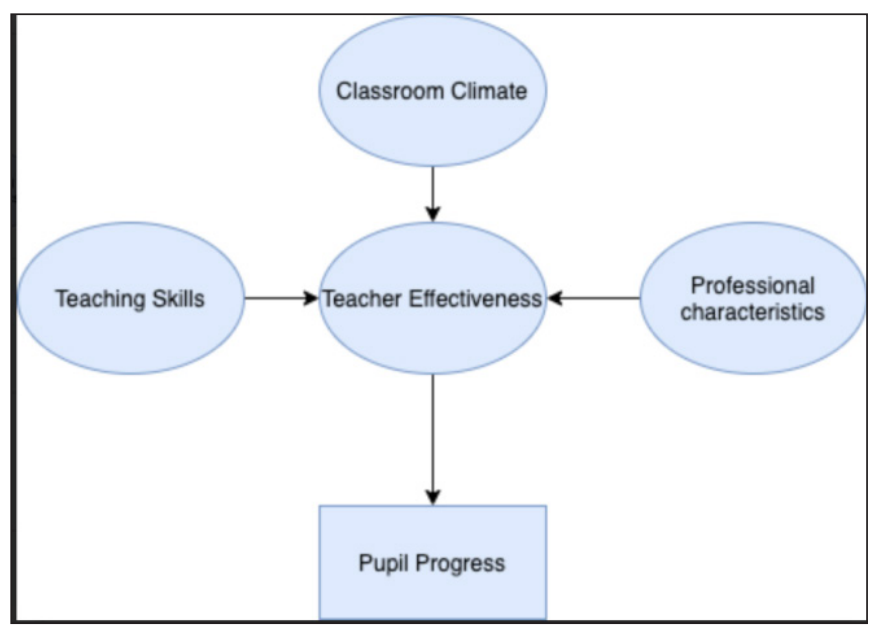

Fig. 1: McBer's model of teacher effectiveness

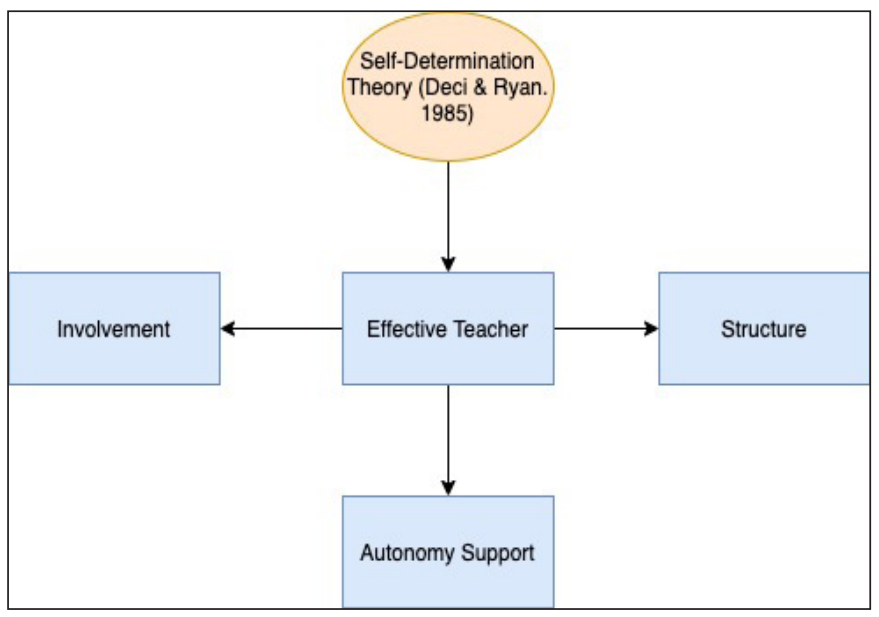

Fig. 2: Need Supportive Teaching Framework

Most of these researches, however, have been conducted in individualistic cultures. In more collectivistic cultures, researches have come up with more mixed findings, especially in India, where controlling demeanor has been shown to both increase performance (Tripathi et al. 2016), and to decrease it, as well (Areepattamannil et al. 2011).

The inquiry remains whether an autonomy-granting tone of voice will enhance students' classroom performance, make them more dedicated to their work, and improve the teachers' effectiveness in increasing students' well-being? We focused on examining these facets by exploring the impact of prosody on the effectiveness of teaching. There are two questions that orient this research:

Research Question 1: If the prosody used by a teacher changes, what impact does it have on intrinsic motivation of students along with its related variables of task valuing, perceived choice, perceived competence, effort expended, and the felt tension?

Research Question 2: Is students' creative problem-solving ability impacted by the type of prosody used?

\section{METHOD}

\section{Sample}

The sample, recruited using convenience sampling, consisted of 93 undergraduate students pursuing various courses (e.g., Hotel management, Maths, Statistics, English) from colleges across Delhi, India (such as the colleges under University of Delhi; Delhi Institute of Hotel Management), in the age group 17-23 years (mean age $=19.27, \mathrm{SD}=1.25$ ). 49 females and 44 males, from low to high middle-class background, with fluency in spoken English, participated in the study.

\section{Design}

The study had a quasi-experimental, between-subject design, that included two experimental groups (autonomy granting and controlling prosody) and one control group (neutral prosody). Follow-up interviews were also conducted with a subset of students.

\section{Measures}

We used The Intrinsic Motivation Inventory (IMI) (Deci 
\& Ryan, 2005) and the Passi-Usha Test of Creative Problem Solving (PUTCPS) (Passi \& Kumar, 1996, as cited in Kohser, 2006), for the present study. The IMI (Deci \& Ryan, 2005) is a 37 item, 7-point Likert-type scale. The response for each item ranges from "not true at all" to "very true". It consists of seven subscales- enjoyment/ interest, value/usefulness, perceived competence, effort, felt pressure and tension, perceived choice, and relatedness, out of which we used the first six subscales for our research. The overall scale is internally consistent (alpha $=0.85$ ), and also shows high discriminant and face validity (Ostrow \& Heffernan, 2018).

The PUTCPS (Passi \& Kumar, 1996, as cited in Kohser, 2006) identifies creative talent among children and adults. It is a subjective test that includes $7 \mathrm{item}$ problems to which non-verbal responses have to be drawn. It assesses the development of critical, creative (originality and elaboration), and integrative thinking skills. The test-retest reliability of the full scale (0.85), the originality dimension (0.74), and the elaboration dimension (0.86) is high. The concurrent validity, which was measured by correlating it with Passi test of creativity (0.40) and Torrance test of creativity (0.56), also lies in the upper range.

\section{Procedure}

\section{Developing the instructions}

Based on the literature review (Haimovitz and Corpus, 2011), we selected a hidden pictures puzzle and a hiddenwords puzzle for the present research. We ensured that the semantics of the instructions were kept as neutral and similar as possible, and they only differed in their acoustic characteristics (Table 1). To test for significant differences between the instructions, and to determine that they were actually conveying what they were meant to convey, i.e., autonomy granting tone, controlling and neutral tone of voice, they were pilot tested for perceived differences between the prosodies on a group of undergraduates $(n=5)$, where they were asked to rate on two 5-point Likert-type rating scales, how pressurizing or supporting they found the researcher's instructions to be. Further, Friedman's non-parametric test revealed that there were significant differences between these three sets of instructions.

Table 1: Acoustic characteristics of different prosodies

\begin{tabular}{llll}
\hline & $\begin{array}{l}\text { Pitch } \\
(\mathbf{H z})\end{array}$ & $\begin{array}{l}\text { Intensity } \\
(\mathbf{d b})\end{array}$ & $\begin{array}{l}\text { Speech rate } \\
\text { (words/second) }\end{array}$ \\
\hline Hidden Pictures Puzzle & & & \\
Autonomy-granting & 44100 & 86.0 & 2.63 \\
Neutral & 32000 & 90.1 & 2.89 \\
Controlling & 22050 & 90.7 & 3.61 \\
Hidden Words Puzzle & & & \\
Autonomy-granting & 44100 & 85.3 & 2.19 \\
Neutral & 32000 & 89.9 & 2.98 \\
Controlling & 44100 & 91.3 & 3.79 \\
$\begin{array}{l}\text { Intrinsic motivation } \\
\text { inventory }\end{array}$ & & & \\
Autonomy-granting & 44100 & 88.4 & 2.37 \\
Neutral & 32000 & 89.2 & 2.38 \\
Controlling & 22050 & 90.5 & 3.15 \\
Passi-Usha Test of & & & \\
Creative problem solving & & & \\
Autonomy-granting & 44100 & 86.5 & 2.38 \\
Neutral & 32000 & 88.9 & 2.55 \\
Controlling & 22050 & 91.6 & 3.19 \\
\hline
\end{tabular}

\section{Effect of prosody on students}

Multiple conductions in groups of 5-15 students were held, with edible incentives for them to participate, in the study. Two sessions were conducted with each group; conduction time with each group was an average of one and a half hours, with a five-minute break in between. In the first session, we informed the participants about their ethical rights, and gave them instructions to complete the hidden pictures puzzle and the hidden words puzzle. In the second session, we administered the creative problem-solving task (Passi \& Kumar, 1996, as cited in Kohser, 2006) and the IMI (Deci \& Ryan, 2005).

We followed this up with telephonic semi-structured interviews with three participants from each group, and this selection was based on convenience and availability. Probes into their reasons for participating in the study, 
their subjective experiences, and their perceived autonomy, were recorded.

\section{RESULTS AND DISCUSSION}

\section{Quantitative Results}

We analyzed the data using one-way ANOVA on SPSS v16.0, testing for significant differences in measures of teaching effectiveness across the three groups. The effect size (eta-squared) was also calculated for each variable.

The descriptive statistics on the variables of Interest are presented in Table 2. As can be seen, on intrinsic motivation, perceived choice, and creative problem solving, the group exposed to the autonomy-granting prosody scored the highest, while the participants in the controlling prosody group scored maximum on task value and tension felt. Highest scores on perceived competence and effort expended were achieved by the neutral prosody group. These statistics are interpreted in more detail below.

Research Question 1: If the prosody used by a teacher changes, what impact does it have on intrinsic motivation of students and its related variables of tension, task valuing, perceived competence, perceived choice, and effort expended?

Table 3 shows that the participants in the three groups did not seem to significantly differ from each other on intrinsic motivation or any other variables related to the same $(p>0.05)$. The effect size values were also in the range from small to medium (Watson, 2019), suggesting that these constructs were not strongly impacted by the independent variable, i.e., the prosody. The maximum impact prosody had was on perceived choice of the participants, where it accounted for $5.4 \%$ variance, while it accounted for only $0.2 \%$ variance in their perceived competence.

Research Question 2: Is students' creative problem-solving ability impacted by the type of prosody used?

Post hoc analysis revealed that the autonomy-granting group did significantly better than the neutral prosody group $(\mathrm{F}=6.01, \mathrm{p}<0.05$, mean difference $=-19.93, \mathrm{p}<0.05)$ on creativity. In terms of its sub-dimensions, the same pattern was found for the scores on elaboration $(F=5.77$, $\mathrm{p}<0.05$, mean difference $=-18.37, \mathrm{p}<0.05)$, while no significant differences were found in originality scores $(\mathrm{F}=2.65, \mathrm{p}<0.05)$. The effect size, however, was in the low range for all three variables (Watson, 2019), suggesting that even though prosody did impact the creativity of participants, it only accounted for around $1 \%$ variance.

\section{Interview Analysis}

We content analyzed the interviews of the participants and calculated a frequency count for responses to each question.

As can be seen from Table 7, most participants had positive views about the use of recorded instructions.

Table 2: Mean scores and SD of participants belonging to autonomy-granting, controlling, and neutral prosody

\begin{tabular}{|c|c|c|c|c|c|c|}
\hline \multirow{2}{*}{ Variable } & \multicolumn{2}{|c|}{ Autonomy-Granting } & \multicolumn{2}{|c|}{ Controlling } & \multicolumn{2}{|c|}{ Neutral } \\
\hline & $\mathbf{M}$ & SD & $\mathbf{M}$ & SD & $\mathbf{M}$ & SD \\
\hline Intrinsic Motivation & 5.86 & 0.99 & 5.68 & 1.09 & 5.48 & 1.12 \\
\hline Perceived Choice & 5.28 & 0.93 & 5.01 & 1.03 & 4.68 & 1.11 \\
\hline Perceived competence & 4.28 & 1.14 & 4.38 & 1.18 & 4.41 & 1.23 \\
\hline Tension & 2.26 & 1.65 & 2.74 & 1.09 & 2.29 & 0.96 \\
\hline Creativity & 59.27 & 25.01 & 46.48 & 22.70 & 39.33 & 19.69 \\
\hline Originality & 9.70 & 2.10 & 9.24 & 2.34 & 8.13 & 3.52 \\
\hline Elaboration & 49.57 & 24.55 & 37.24 & 21.68 & 31.20 & 17.18 \\
\hline
\end{tabular}

Note: $n=93$. Autonomy granting $(n)=30$, Controlling $(n)=33$, Neutral $(n)=30$. 
Table 3: ANOVA results comparing the autonomy-granting, controlling, and neutral prosody groups on their intrinsic motivation and related variables

\begin{tabular}{|c|c|c|c|c|c|c|}
\hline Variable & Source of Variation & SS & MS & F value & $p$ value & Effect size $\left(\eta^{2}\right)$ \\
\hline \multirow[t]{2}{*}{ Intrinsic motivation } & Between group & 2.12 & 1.06 & 0.92 & 0.40 & 0.02 \\
\hline & Total & 105.63 & & & & \\
\hline & Within group & 145.93 & 1.621 & & & \\
\hline & Total & 146.69 & & & & \\
\hline Perceived choice & Total & 100.60 & & & & \\
\hline \multirow[t]{3}{*}{ Perceived competence } & Between group & 0.288 & 0.14 & 0.10 & 0.90 & 0.00 \\
\hline & Within group & 126.27 & 1.40 & & & \\
\hline & Total & 126.56 & & & & \\
\hline Effort expended & Between group & 0.44 & 0.22 & 0.13 & 0.88 & 0.00 \\
\hline Tension felt & Total & 148.79 & & & & \\
\hline
\end{tabular}

Note: $d f($ between group $)=2, d f($ within group $)=90, d f($ total $)=92$.

Table 4: ANOVA results comparing the autonomy-granting, controlling, and neutral prosody groups on their creative problem solving

\begin{tabular}{lllllll}
\hline Variable & Source of Variation & SS & MS & F value & p value & Effect size $\left(\eta^{2}\right)$ \\
\hline Creativity & Between group & 6128.79 & 3064.40 & 6.01 & $0.00^{*}$ \\
& Within group & 45878.78 & 509.76 & & 0.12 \\
Originality & Total & 52007.57 & & & 0.08 \\
& Between group & 39.08 & 19.54 & 2.65 & & \\
& Within group & 663.83 & 7.38 & & $0.00^{*}$ \\
Elaboration & Total & 702.90 & & & 0.11 \\
& Between group & 5270.05 & 2635.03 & 5.77 & & \\
& Within group & 41090.23 & 456.56 & & & \\
& Total & 46360.28 & & & & \\
\hline
\end{tabular}

${ }^{*} p<0.05$. Note: $d f($ between group $)=2, d f($ within group $)=90, d f($ total $)=92$.

Table 5: Perception of the instructions by the participants

\begin{tabular}{|c|c|c|c|c|}
\hline Theme & Responses & Autonomy Granting & Controlling & Neutral \\
\hline Use of Recorded Instructions & Positive views & 3 & 3 & 2 \\
\hline Difference from face-to-face teaching & Better & 2 & 1 & 2 \\
\hline \multirow[t]{2}{*}{ Artificiality of Instructions } & Natural & 3 & 2 & 2 \\
\hline & Artificial & 1 & 1 & 1 \\
\hline Connection felt to the Instructor & Yes & 1 & 0 & 0 \\
\hline Importance given to tone of voice & Minimal & 2 & 2 & 3 \\
\hline
\end{tabular}


They mentioned that it helped them concentrate better and removed distractions that are associated with faceto-face teaching. In spite of this, they could not form an emotional bond with the "virtual" teacher, and were not much impacted by the tone in which the instructions were given to them.

Overall, the students in all three conditions positively viewed the tasks as fun activities, easy and valuable (Table 6). However, they felt that their solutions to the items in PUTCPS (Passi \& Kumar, 1996, as cited in Kohser, 2006) were not good enough to be implemented. Those in the autonomy-granting group differed from others; in that, they thought they had more independence in coming up with solutions, and were thinking more pro-socially when trying to come up with ideas.

As reported in the results, pre-recorded instructions were well received by most of the participants, but despite this, the results of the present study varied from what was shown previously by Weinstein et al. (2017, 2019).

The participants were all intrinsically motivated to participate, were mostly tension free during the study, and put in effort while performing the tasks. Furthermore, while the students in the autonomygranting and controlling group were in a pleasant mood while doing the activities, those in the neutral prosody group were in a more negative and serious mood (Table $7)$.

\section{What could be the plausible reasons for these compelling findings?}

It could be the general lack of concern for prosody as perceived by the students in our sample. Seven out of the nine participants reported being more focused on

Table 6: Perceptions of the tasks and activities by the participants

\begin{tabular}{|c|c|c|c|c|}
\hline Theme & Responses & Autonomy Granting & Controlling & Neutral \\
\hline \multirow[t]{2}{*}{ Perception of tasks } & Fun & 3 & 3 & 3 \\
\hline & Work & 0 & 0 & 2 \\
\hline \multirow[t]{2}{*}{ Perceived difficulty level of tasks } & Mediocre & 3 & 3 & 2 \\
\hline & Hard & 0 & 0 & 1 \\
\hline \multirow[t]{2}{*}{ Benefits and downsides of tasks } & Many benefits & 3 & 2 & 2 \\
\hline & Time-taking & 1 & 0 & 0 \\
\hline $\begin{array}{l}\text { Independence in coming up with } \\
\text { solutions }\end{array}$ & No & 0 & 2 & 2 \\
\hline Probability of idea implementation & Low & 3 & 3 & 3 \\
\hline \multirow[t]{2}{*}{ Pro-social orientation in solutions } & High & 3 & 1 & 1 \\
\hline & Low & 0 & 2 & 2 \\
\hline
\end{tabular}

Table 7: Personal affect and cognitions of participants

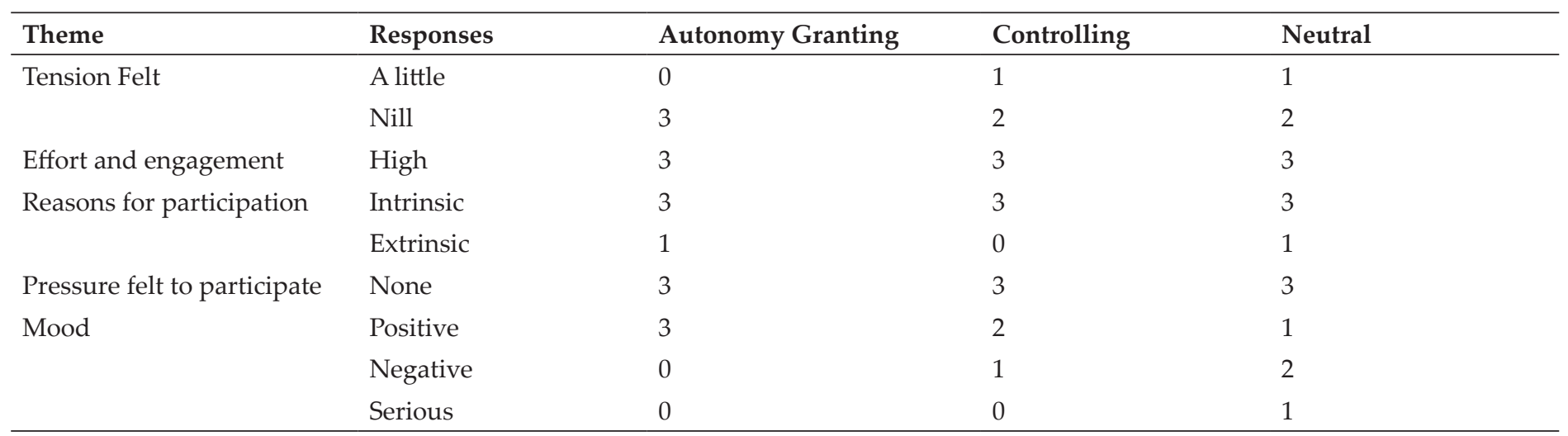


the words than the tone of voice, indicating passive listening. An interviewee in the controlling condition, for instance, reported that "Just the words had an impact on me, the tone did not matter". However, research done previously has found that to create a difference, the listeners need to be attentive to the verbal cues of speech (Paulmann et al. 2019). Moreover, even though all students had positive views about the use of prerecorded instructions, majority of them ( 8 out of 9) reported not having experienced any connection with the instructor, which is necessary if the speaker wants to create any considerable impact on the listeners (Roth \& Assor, 2012). Research has also shown that communication of emotions and motivation differ from each other. A teacher could be using an autonomysupportive tone, but may still seem overpowering to her students (Zougkou et al. 2017). Since we did not vary emotional expression, this could be a possible reason for no substantive difference. These findings raise concerns regarding how effective prosody varies across cultural domains, in creating an impact on individuals. Even though no data on the current participants' cultural background was noted, it may be pertinent to remark that our sample chosen were from central universities in India, where students from all over the country enroll. By virtue of this aggregation, they do represent varied cultural contexts. Therefore, it may be apt to surmise that prosody may not have a significant role to play in the present Indian cultural context!

To capture how the participants diversity, or the lack thereof, could have contributed to the earnest findings, the related constructs of selection bias and confirmation bias can be explored. Confirmation bias occurs when an individual favors information that confirms their pre-existing beliefs. Selection bias refers to when proper randomization is not used in the selection of the participants of a study, and so the sample shares some common characteristics which affect the results. We conducted this study on students studying in New Delhi, India, irrespective of whichever culture they originally came from. The education system in the capital of India, is structured in such a way that students are accustomed to a certain degree of control and do accord it a status of a 'norm'. Because of this, they may not have responded positively to autonomy-granting prosody. On the contrary, this conditioning accentuates the students to find a controlling prosody motivating to them! Besides, there is no previous research evidence to throw light on prosody as significantly impacting teaching effectiveness. Factors such as presentation style, subject knowledge, maintaining discipline and decorum, and showing responsibility for student failure contribute more to the value of a teacher in India (Bambawale et al. 2018; Barman \& Bhattacharyya, 2017).

The setting of the study may also have contributed to the results. The fact that it was a curated situation in which we dictated what the participants had to do, might have possibly overridden the impact of prosody on the students' levels of autonomy and intrinsic motivation. Furthermore, previous research has demonstrated that under laboratory conditions, it is more difficult to escape from a controlling message as it tends to hold more attentional resources, while autonomous messages may be discounted if they are not self-relevant (Zougkou et al. 2017). As the activities we used were self-explanatory, it is possible that participants in the autonomous condition did not pay much attention to the instructions, while controlling instructions may still have been attended to, because of their inherent nature.

\section{What causes creativity to be enhanced under autonomous conditions?}

Interestingly, even though the prosody had no impact on other variables, the autonomy-granting tone did enhance participants' creativity $(\mathrm{F}=6.02, \mathrm{p}<0.05)$. One of the many reasons that have been rendered, is that when the psychological needs of an individual are met under an autonomy-granting environment, it enhances their constructive tendencies. This is in line with the Theory of Self-Determination (Deci \& Ryan, 1985), positing that autonomy enhances the intrinsic motivation of individuals, which further improves their creativity and well-being.

An autonomy-granting tone may have put the participants in a favorable mood, which mediates the relationship between autonomy and creative problem solving (Weinstein et al. 2019). Autonomy allows individuals to get in touch with their true self and 
enhances their focus and attention on the task at hand (Gillet et al. 2012). Positive emotions, in turn, build cognitive resources which are adaptive, and thus lead to higher performance, as given by The Broaden and Build Theory. The interview responses support this understanding. In the autonomy-granting condition, all three participants reported experiencing a positive mood during the study, whereas participants in the neutral prosody group reported a more negative and serious mood. To quote one student, "I was in a serious mood and trying to do the task with my 100\%. I was serious and focused. After it was over, I was relieved that I could get it done."

Since the items in the tasks were ill-defined and infinite solutions could be generated, autonomy may have also made the students feel independent in coming up with accurate and feasible solutions to the problems (Nair \& Alkiyumi, 2011). Participants in the autonomy-granting condition stated, "I felt free to come up with any solutions I wanted, based on the situation. There were no restrictions, we could use colored pens, everything was free. I loved it, I drew whatever I could." Individuals in the neutral condition, however, reported, "I had some problems, like, what to draw, what solution has to be there, I didn't feel I was doing it correctly."

It has also been found that when exposed to autonomygranting prosody, individuals' pro-social tendencies are enhanced (Grant \& Berry, 2011), which leads to greater creativity, and more elaborate and useful solutions, finds support in our interview. All three participants in the autonomy-granting condition said that their solutions would help other people, while only one participant in the neutral condition said so, the other two did not feel this way. To quote, "The solutions I came up with were not very great. So, I didn't do them with the purpose of helping other people."

A noteworthy finding is that participants' creativity in the autonomy-group was higher not because they gave more original responses, but because they elaborated more on them. The fact that participants could not implement the solutions they came up with, could have reduced the originality of their ideas. As the interview responses reveal, all participants had low expectations about their ideas getting implemented. The setting of the experiment, and the kind of incentives that were offered to the participants in the study, may have accounted for such findings. Giving explicit rewards for an activity leads to a reduction in originality as compared to when non-contracted for rewards, or no rewards are given. Since the participants were offered chocolates as a token of their participation, and this was informed to them beforehand, it could have led to a reduction in the originality of ideas.

\section{Implications}

The present research gives insight into how a teacher can achieve positive outcomes such as building the intrinsic motivation of students, enhancing their competence, and reducing stress. We found that one way to do so, is to modulate the voice while giving instructions. Instructors should use an autonomy-granting tone whenever possible, to enhance their students' divergent thinking and problem-solving capacity which subscribes to the need-supportive teaching framework. Moreover, before using an autonomy-granting tone, the teacher must explain to the students the concept of tone of voice, and the tactics she would be using to engage the students, so that passive listening does not occur. This does not mean that a neutral or a controlling tone should never be used by a teacher. Depending on the context and the task at hand (e.g.- to deal with indiscipline), an instructor can sometimes shift to a neutral or controlling prosody. In addition, as the need supportive framework advances, it is equally important to involve students in the classroom activities. The present research suggests that this can be done by conveying liking and care towards the students. Other suggestions to create a positive classroom, in line with what McBer posits, include highlighting the positive and fun aspects of tasks, connecting classroom activities and homework to long-term goals of students, and using more problemsolving activities, can be folded into the daily teaching curriculum. Teachers should also control the tasks' level of difficulty according to the students' skills, discuss practical applications, and implement the ideas students come up with, even if only at the classroom level. 
In addition to teaching and education, the results of this study have implications for workers in the fields of counseling and advocacy. Counselors and psychotherapists should especially keep in mind the prosody they are using with their client, as it can impact the client's emotional state during the counseling sessions, the quality of the therapeutic relationship, and the effort client puts into bringing about change. The prosody used also influences advocates, whose jobs are to convince other people through what they say. They must learn which tone to use under which condition in order to create a longer lasting effect on listeners.

\section{CONCLUSION}

We explored the impact of motivational prosody on teaching effectiveness, using a quasi-experimental study on ninety-three undergraduate students. Teaching effectiveness was measured through students' intrinsic motivation, task valuing, perceived choice, perceived competence, tension, effort expended, and creative problem solving. The prosodies investigated included autonomy-granting, controlling, and neutral prosodies. Statistical analysis along with the follow-up interviews revealed that the groups significantly differed in creative problem solving, with an effect size of 0.118 . Moreover, the difference in scores for creative problem solving was due to differences in level of elaboration by participants, whereas the originality of responses was same across groups. This suggests that even though prosody has a role to play, a much more complex network of interconnected factors determines the student-teacher relationship. The results have wide ranging implications for teachers, lawyers, and counselors.

\section{ACKNOWLEDGMENTS}

Many thanks go to the professors of the psychology department from Lady Shri Ram College, and Shubhi ma'am from Delhi Institute of Hotel Management, for their constant support and guidance.

\section{REFERENCES}

Areepattamannil, S., Freeman, J.G. and Klinger, D.A. 2011. Intrinsic motivation, extrinsic motivation, and academic achievement among Indian adolescents in Canada and India. Social Psychology of Education, 14(3): 427-439.
Bambawale, M., Hughes J. and Lightfoot, A. 2018. Exploring teacher evaluation processes and practices in India: A case study (Report No. 978-0-86355-890-0). New Delhi: British Council.

Barman, P. and Bhattacharyya, D.D. 2017. Job Satisfaction of Teacher Educators in Different Types of B.Ed. Colleges in West Bengal. IOSR Journal of Humanities and Social Science, 22(02): 80-99.

Deci, E.L. and Ryan, R.M. 1985. Conceptualizations of Intrinsic Motivation and Self-Determination. Intrinsic Motivation and Self-Determination in Human Behavior, pp. 11-40. doi:10.1007/9781-4899-2271-7_2

Deci, E.L. and Ryan, R.M. 2005. Intrinsic Motivation Inventory (IMI).[WWW document]. Self-Determination Theory. Retrieved from http://www. selfdeterminationtheory. org/ intrinsic-motivation-inventory

Gillet, N., Vallerand, R.J., Lafrenière, M.K. and Bureau, J.S. 2012. The mediating role of positive and negative affect in the situational motivation-performance relationship. Motivation and Emotion, 37(3): 465-479.

Grant, A.M. and Berry, J.W. 2011. The Necessity of Others is The Mother of Invention: Intrinsic and Prosocial Motivations, Perspective Taking, and Creativity. Academy of Management Journal, 54(1): 73-96.

Haimovitz, K. and Corpus, J.H. 2011. Effects of person versus process praise on student motivation: stability and change in emerging adulthood. Educational Psychology, 31(5): 595-609.

Kohser, S. 2006. A Factor-Analytic Study of Intuition and Mental Abilities and its Educational Implications (Doctoral Dissertation, Aligarh Muslim University). Retrieved from http://hdl.handle. net/10603/52778

McBer, H. 2012. Research into teacher effectiveness : A model of teacher effectiveness (Number 216). Great Britain: Department for Education and Employment.

Nair, S. and Alkiyumi, M. 2011. Investigation the Relationship between Intrinsic Motivation and Creative Production on Solving Real Problems. Sosiohumanika, 4(2): 185-196.

Paulmann, S., Weinstein, N. and Zougkou, K. 2019. Now listen to this! Evidence from a cross-spliced experimental design contrasting pressuring and supportive communications. Neuropsychologia, 124: 192-201.

Roth, G. and Assor, A. 2012. The costs of parental pressure to express emotions: Conditional regard and autonomy support as predictors of emotion regulation and intimacy. Journal of Adolescence, 35(4): 799-808.

Tripathi, R., Savani, K. and Cervone, D. 2016. Are Motivational Effects of Autonomy Universal? Contrasting Results among Indians and Americans. Academy of Management Proceedings, (1): 13051. 
Weinstein, N., Vansteenkiste, M. and Paulmann, S. 2019. Listen to your mother: Motivating tones of voice predict adolescents' reactions to mothers. Developmental Psychology, 55(12): 25342546.

Weinstein, N., Zougkou, K. and Paulmann, S. 2017. You 'have' to hear this: Using tone of voice to motivate others. Journal of Experimental Psychology: Human Perception and Performance, 44(6): 898-913.
Zougkou, K., Weinstein, N. and Paulmann, S. 2017. ERP correlates of motivating voices: quality of motivation and time-course matters. Social Cognitive and Affective Neuroscience, 12(10): 1687-1700. 